\title{
Nanocrystal Synthesis within Solid-State Confinement
}

ISSN: 2578-0255

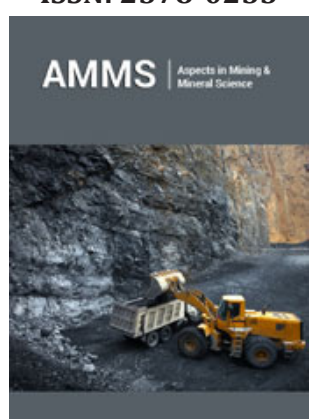

*Corresponding author: Ki-Wan Jeon, Department of Engineering in Energy and Applied Chemistry, Silla University, Busan 46958, Korea

Submission: 侮July 24, 2020

Published: 侮August 14, 2020

Volume 5 - Issue 3

How to cite this article: Ki-Wan Jeon. Nanocrystal Synthesis within Solid-State Confinement. Aspects Min Miner Sci. 5(3). AMMS. 000612. 2020.

DOI: 10.31031/AMMS.2020.05.000612

Copyright@ Ki-Wan Jeon, This article is distributed under the terms of the Creative Commons Attribution 4.0 International License, which permits unrestricted use and redistribution provided that the original author and source are credited.

\section{Ki-Wan Jeon*}

Department of Engineering in Energy and Applied Chemistry, Silla University, Busan, Korea

\begin{abstract}
Designing, synthesizing, and modifying nanomaterials (below $100 \mathrm{~nm}$ in size) are at the leading edge of emerging nanoscience and nanotechnology due to exhibiting a wide range of enhanced and new physicochemical properties such as mechanical, thermal, electronic, optical properties which are significantly different from their bulk counterparts. It makes these materials superior and indispensable in many modern technological applications. By taking advantage of the unique physicochemical properties of nanocrystals, different synthetic strategies, bottom-up and top-down approach, have been developed. Among those synthetic methods, solution-based process to synthesize nanocrystals have been mostly employed with a specific precursor, stabilizing and/or capping agent. Despite remarkable advances in wetchemical syntheses for precisely synthesizing and tailoring nanocrystals, solution-based procedures are still restricted to preparing a wider variety of nanocrystals owing to limited available precursors, organic surfactants, and reaction conditions. Unlike well-established solution-based process, solid-state reaction, which allows us to synthesize almost unrestricted metal-combinations and alloys, has been scarcely employed to synthesize nanocrystals due to inevitable sintering and aggregation during the conventional solid-state reaction process required high-temperature $\left(>300{ }^{\circ} \mathrm{C}\right)$. Recently, high-temperature solidstate reaction with different type of templates for synthesizing nanocrystals has been devised, which could provide a potential novel nanoparticles not synthesized via conventional solution-based chemical reaction. This review will summarize syntheses of various nanocrystals through high-temperature solidstate reaction with spatial confinement strategy. The discussion will develop to the crucial examples of synthesizing nanocrystals through solid-state reaction pathway, which involves growing crystals spatially restricted within thermally stable porous materials, nanoparticles, or two-dimensional substrates.
\end{abstract}

Keywords: Nanocrystals; Nanomaterials; Morphological; Silica

\section{Introduction}

Due to wide ranging technological applications and intriguing physicochemical properties, synthesis of nanocrystals (NCs) has been immensely active area of research. The properties in nanoscale are severely affected by the architecture and compositions of the NCs and these parameters can be tailored by understanding the complex nanostructure-growth mechanisms and their precise engineering. To date, solution-based methods, generating nanostructures with variable dimensionality and complexity have been significantly progressed, which require rigorous control of reaction-conditions with the use of surfactants and difficulty in synthesizing multicomponent-NCs with incompatible interfaces [1]. To synthesize multicomponent-NCs, other approaches through a process of chemical etching, galvanic chemistry, ion-exchange, with atomic diffusion-mediated nanoscale Kirkendall effect to synthesize multicomponent-NCs have been employed [2,3]. Recently another strategies by utilizing variety of hollow and confined nanostructures have been explored, giving rise to chemical reactions and growing metal NCs within their well-protected confined environment, high surface area, controllable interior access, and solution-partition [4,5]. These, the 'rattle' or 'yolk-shell' nano-structures where small NCs are confined within a hollow-porous shell have been intensively investigated [6-9]. Due to tedious multistep processes of the aforementioned approaches, research groups have employed ship-in-a-bottle method, producing NCs inside hollow silica microsphere [10-13]. Although traditional high-temperature solid-state reaction route allows 
us to synthesize unrestricted metal combinations and alloys, the high-temperature synthesis has been rarely explored synthesizing multimetallic NCs on the exposed surface due to the facile sintering of NCs [14-19]. In this review, I highlight syntheses of NCs within spatially confined reaction medium via high temperature solid-state reaction routes. For this particular reaction, vital to the strategy is the 'thermally isolated environment' of silica medium during the high temperature reactions among diverse precursor-metal ions or $\mathrm{NCs}$, controlling their migration, sintering and phase-mixing/segregation. Herein, I will introduce crucial examples of high-temperature nanoconfined solid-state reaction.

\section{Synthesis of Nanocrystals within Spatially Confined} Reaction Medium through High Temperature SolidState Reaction

In order to apply high temperature solid-state reactions to the synthesis of nanocrystals, tens of nanometer-sized silica nanosphere as the reaction medium was employed, which can allow for treatment of the high-temperature reactions among the medium-embedded atoms, ions and/or nanoparticles. Through this strategy, various chemical processes such as the diffusion and movement, morphological transformations and solid-state chemical reactions can be confined within nanoscale and be systemati- cally investigated. The ultimate goal of these strategies is to expand the scope and utility of the NC-synthesis beyond the conventional solution-based reactions and to fully exploit the diverse combinations of reactants to generate libraries of nanocrystals. Shin et al. [20] produced $\mathrm{Fe}_{3} \mathrm{O}_{4} / \mathrm{PdO}$ heterodimer encapsulated with silica nanosphere by air annealing silica nanospheres $(\sim 30 \mathrm{~nm})$ encapsulated with $\mathrm{Fe}_{3} \mathrm{O}_{4} \mathrm{NCs}$ and $\mathrm{Pd}^{2+}$ complexes at $800{ }^{\circ} \mathrm{C}$, it can be further transformed into either a FePd alloy or $\mathrm{Fe}_{3} \mathrm{O}_{4} / \mathrm{Pd}$ hybrid NCs by second heat-treatment [20]. When Au component was added to the aforementioned system, ternary system of FeAuPd NC within $\mathrm{SiO}_{2}$ nanosphere (FeAuPd@SiO 2 ) was synthesized at the temperature higher than $700{ }^{\circ} \mathrm{C}$ under $4 \% \mathrm{H}_{2} / 96 \% \mathrm{~N}_{2}$ atmosphere. This reductive heat-treatment caused outward migration of FeAuPd NC, leaving behind a hole inside silica (Figure 1) [21]. Additionally, subsequent air-annealing of FeAuPd@SiO nanomaterials resulted into the phase-segregated oxidized hybrid NCs with different morphologies such as spherical, dumbbell, three-ball-snowman, and mushroom-like structures, depending on the degree of migration of the parent alloy $\mathrm{NC}$ inside the $\mathrm{SiO}_{2}$ nanospheres (Figure 1). The migration of the FeAuPd NCs within the silica could be attributed to the change of amorphous silica-phase into the glass transition phase at high temperatures with accompanying decreasing viscosity of the $\mathrm{SiO}_{2}$ nanosphere.
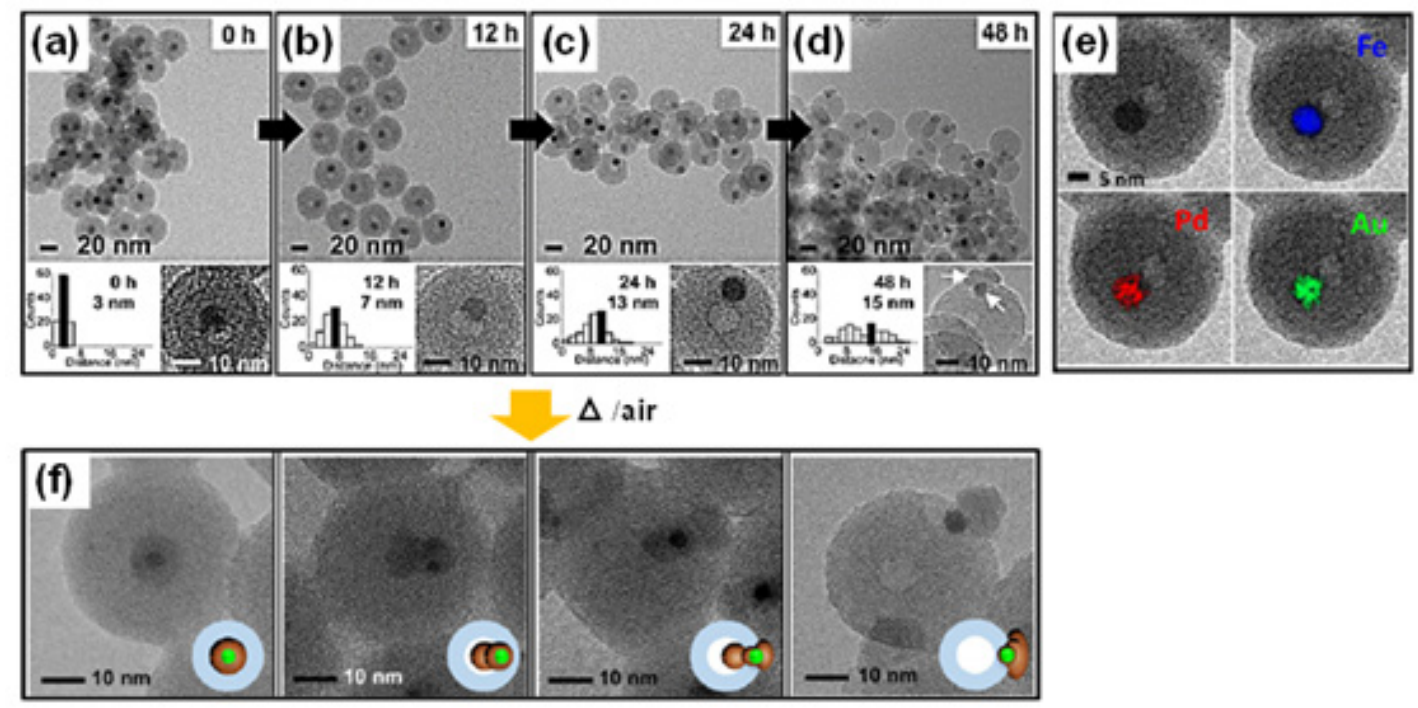

Figure 1: (a-d) TEM and HRTEM images of FeAuPd@ $\mathrm{SiO}_{2}$ at different annealing time, (e) elemental mapping images of the core NCs (TEM-EELS), (f) HRTEM images of morphological change of the core NCs with different position in the $\mathrm{SiO}_{2}$ nanosphere. Adopted with permission from [21].

Thermal transformation of NCs was also investigated by alternative heat-treatment of the silica nanosphere encapsulating both $\mathrm{Au} \mathrm{NC} / \mathrm{Au}^{+}$and $\mathrm{M}^{2+}\left(\mathrm{M}=\mathrm{Pt}\right.$ or $\mathrm{Cu}$ ) (Figure 2). In case of $\mathrm{Au} / \mathrm{Pt}^{2+} @$ $\mathrm{SiO}_{2}$, the resultant $\mathrm{Au} / \mathrm{Pt}^{2+} \mathrm{NCs}$ were thermally converted into a reduced AuPt NCs under reductive environment [22]. Upon oxidative annealing, the $\mathrm{AuCu} \mathrm{NCs}$ inside $\mathrm{SiO}_{2}$ nanospheres produced by reductive annealing at $500{ }^{\circ} \mathrm{C}$ uniquely in-situ transformed to different $\mathrm{Au}-\mathrm{CuO}$ heterostructures with varying either reaction temperature or $\mathrm{Cu}^{2+}$ content [23]. The distinct evolution pathway of $\mathrm{Au} / \mathrm{CuO}$ heterodimer through the kinetically accessed and oxidatively phase-segregated Au@CuO core@shell, reveals the escaping motion of the encapsulated Au-core towards silica surface, which is more facilitated by a thicker $\mathrm{CuO}$ shell (Figure 2). This result also demonstrated the high thermal stability of the Au@CuO with a very thin CuO-shell thickness due to the insufficient compressive lattice strain. This study suggests that morphological thermal stability and operational durability of hybrid NCs under high temperature operation can be adjusted based on the compositional and morpholog- 
ical parameters. Although spatially confined NCs within thermally stable reaction medium can be synthesized via high-temperature solid-state reaction, it is still challenging for synthesis of highly mobile small NCs due to becoming low viscosity of the silica medium at high temperature. And hence, the investigation of their high temperature solid-state behavior and chemistry is difficult (Figure 3). To suppress the mobility of the small clusters within elastic reaction medium at high temperature, silica nanosphere modified with porogenic amino silanes was employed [24]. Such aminated silica medium at high temperature generates internal porosity gradient due to decomposition of the amine-functional groups and the generated internal pores limit the nucleation, growth and migrations of NCs. By taking advantage of this strategy, a diverse pool of NCs with Pd, Pd-metal alloy, Pd-metal oxide hybrids were synthesized with first-row transition ions embedded in the aminated silica medium under different thermal conditions.

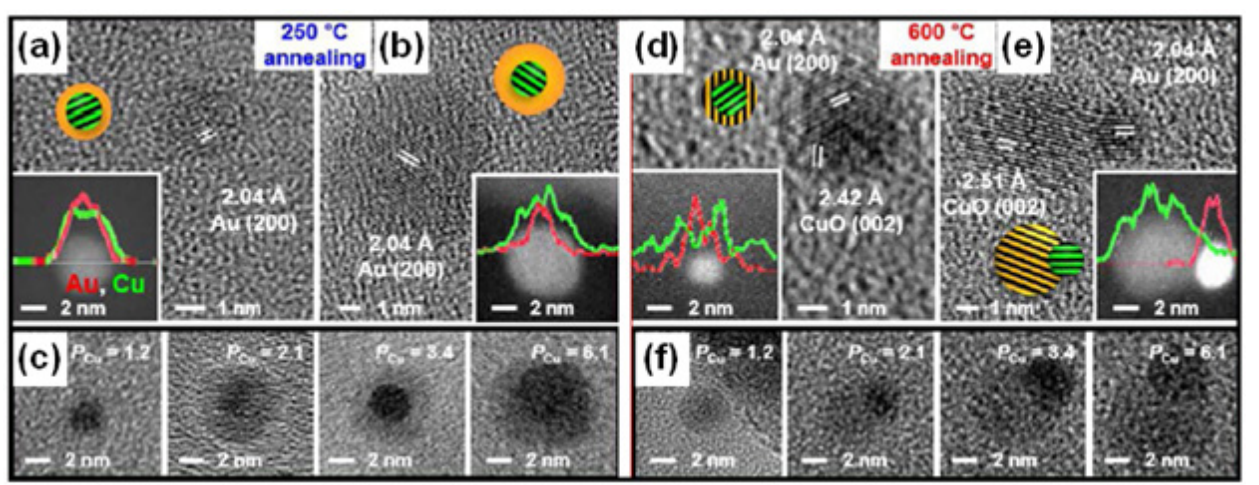

Figure 2: HRTEM images of oxidative heat-treatment of $\mathrm{AuCu}$ core $\mathrm{NCs}$ inside $\mathrm{SiO}_{2}$ nanosphere with different $\mathrm{Cu}$ content and temperature. Adopted with permission from [23].
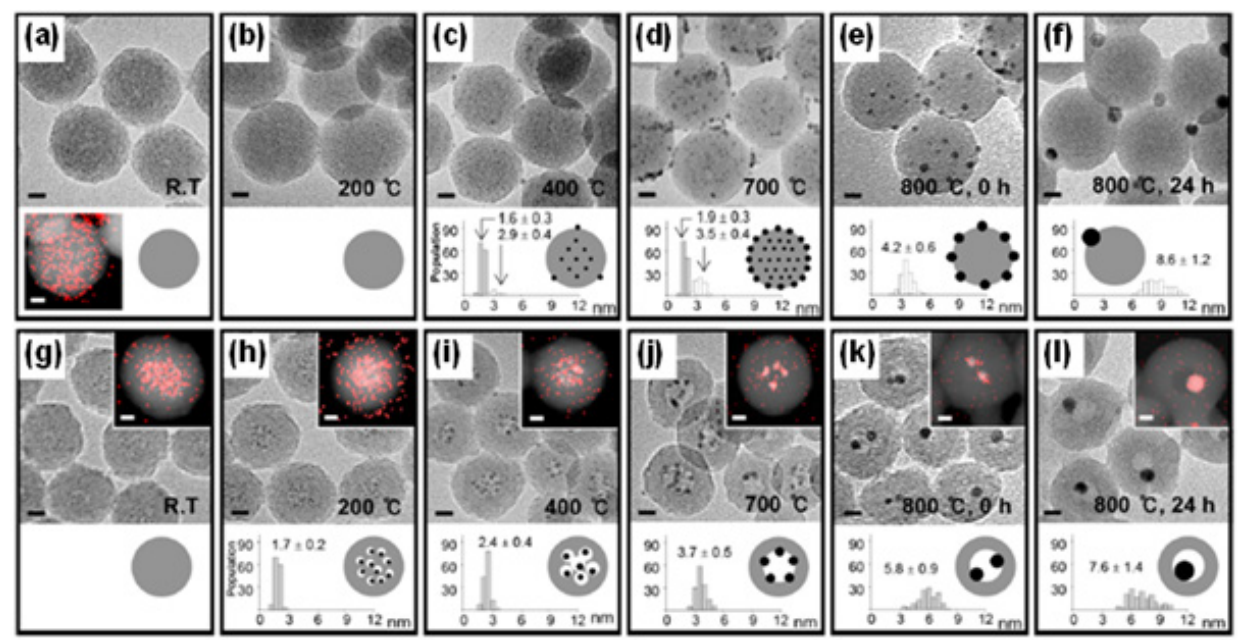

Figure 3: (a-f) HRTEM images of $\mathrm{Pd}^{2+} / \mathrm{SiO}_{2}$ nanoparticles without porogenic amine modification at different annealing temperature, (g-I) HRTEM images and STEM-EDS elemental mapping of $\mathrm{Pd}^{2+} / \mathrm{SiO}_{2}\left(\mathrm{NH}_{2}\right)_{\mathrm{s}}$ nanoparticles with porogenic amine modification at different annealing temperature (scale bar: 10nm). Adopted with permission from [24].

Spatial confinement approaches can transform a structure of the nanomaterial from core@shell into hollow structure, which was conducted with metal cation $\left(\mathrm{Pt}^{2+} / \mathrm{Pd}^{2+}\right)$-incorporating-silica-encapsulated manganese oxide core@shell upon annealing in reductive environment. The morphology of the core@shell structure was gradually transformed into a hollow structure composed of manganese silicate embedded with tiny metal NCs [25]. Additionally, a unique reversible thermal transformation between hollow structure and solid core@shell structure of $\mathrm{Mn}_{3} \mathrm{O}_{4}$ core encapsulated in silica shell incorporating Pd/PdO NCs, upon alternatively thermal treatment under oxidative and reductive environment, respectively (Figure 4) [26,27]. Mechanistically, the hollowing pro- cess of the manganese oxide core could be ascribed to the phase transition of initially separated $\mathrm{MnO}$ and $\mathrm{SiO}_{2}$ into a thermodynamically more favorable silicate phase through outward diffusion of $\mathrm{Mn}^{2+}$ ions toward $\mathrm{SiO}_{2}$ behind the cavity by oxidative thermal annealing. Moreover, the hollow silicate nanospheres reverted to the initial core@shell structure by the filling of the interior cavities with a phase-separated $\mathrm{Mn}_{3} \mathrm{O}_{4}$ NCs. Additional incorporation of $\mathrm{Ni}^{2+}$ in the silica shell resulted in the growth of a single $\mathrm{Ni} \mathrm{NC}$ at the newly generated hollow silicate cavity, producing a yolk@shell structure through a distinct transformation pathway involving preferential reduction of $\mathrm{Ni}$ over $\mathrm{Mn}$ in the in-situ generated $\mathrm{Ni}_{\mathrm{x}} \mathrm{Mn}_{1-\mathrm{x}} \mathrm{O}$ mixed-metal oxide phase [28]. 


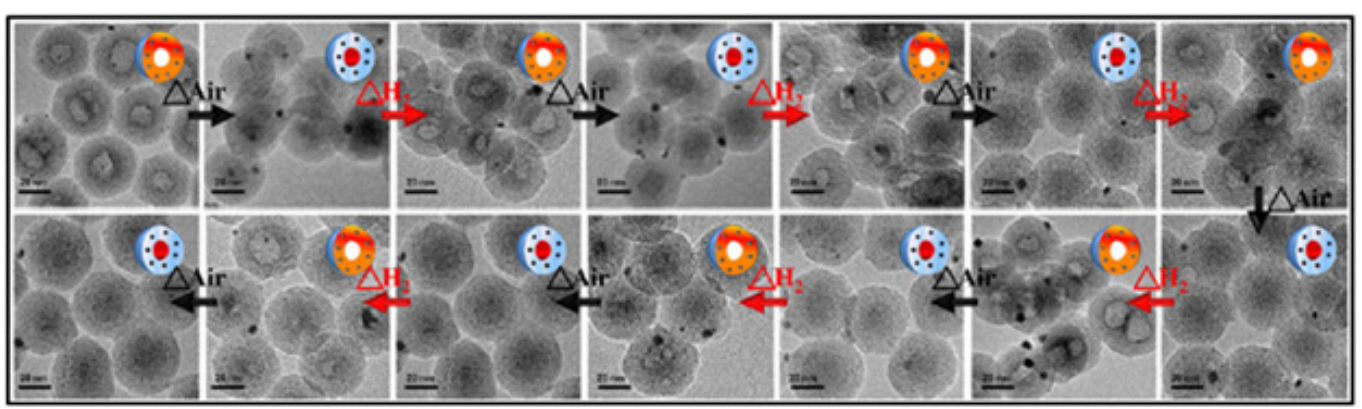

Figure 4: TEM images of switchable morphology between core@shell and hollow structure by alternatively annealing at $500{ }^{\circ} \mathrm{C}$ under oxidative and reductive environment. Adopted with permission from [26].

\section{Conclusion}

Aforementioned strategies could take a place as a very useful tool to synthesize a variety of NCs through high temperature solid-state reaction route. The use of thermally stable reaction medium, silica, containing metal precursors or NCs can provide a platform to investigate various heat-induced processes involving migration, phase segregation/mixing, complex morphological modulation and reversible hollowing, which ultimately provides libraries of surfactant-free NCs. Unlike solution-based reactions, by leveraging the confinement effect and highly viscous-rubbery-state of silica at high temperatures, solid-state reactions can be conveniently captured at multiple stages and complex NC-morphologies can be isolated as synthetic snapshots. Additionally, by generating micro-pores inside silica, thermally-induced migration behavior of highly mobile small sized NCs can be restrained, giving rise to numerous combinations of NCs. The insight accumulated from investigation of the spatial confinement strategies via high-temperature solid-state reaction could provide an opportunity to open new paradigm for the research field in nanotechnology. This could also give a customizable platform for various applications including renewable energy technology, catalysis, bio-imaging, etc.

\section{Acknowledgement}

This work was supported by the National Research Foundation of Korea (NRF) funded by the Ministry of Science, ICT \& Future Planning (MSIP) (NRF-2020R1G1A1007160).

\section{References}

1. Fenton JL, Steimle BC, Schaak RE (2018) Tunable intraparticle frameworks for creating complex heterostructured nanoparticle libraries. Science 360(6388): 513-517.

2. Xia X, Wang Y, Ruditskiy A, Xia Y (2013) $25^{\text {th }}$ Anniversary article: galvanic replacement: a simple and versatile route to hollow nanostructures with tunable and well-controlled Properties. Advanced Materials 25(44): 6313-6333

3. Huang X, Tang S, Yang J, Tan Y, Zheng N (2011) Etching growth under surface confinement: An effective strategy to prepare mesocrystalline Pd nanocorolla. Journal of the American Chemical Society 133(40): 1594615949.

4. Wang X, Feng J, Bai Y, Zhang Q Yadong Y (2016) Synthesis, properties, and applications of hollow micro-/nanostructures. Chemical Review 116(18): 10983-11060.
5. Miszta K, Brescia R, Prato M, Bertoni G, Marras S, et al. (2014) Hollow and concave nanoparticles via preferential oxidation of the core in colloidal core/shell nanocrystals. Journal of the American Chemical Society 136(25): 9061-9069.

6. Sanles Sobrido M, Exner W, Rodríguez Lorenzo L, Rodríguez González B, Correa Duarte M, et al. (2009) Design of SERS-encoded, submicron, hollow particles through confined growth of encapsulated metal nanoparticles. Journal of the American Chemical Society 131(7): 2699-2705.

7. Zhang Q Lee I, Joo JB, Zaera F, Yin Y (2013) Core-shell nanostructured catalysts. Accounts of Chemical Research 46(8): 1816-1824.

8. Chen Y, Chen HR, Shi JL (2014) Construction of homogenous/heterogeneous hollow mesoporous silica nanostructures by silica-etching chemistry: principles, synthesis, and applications. Accounts of Chemical Research 47(1): 125-137.

9. Lian J, Xu Y, Lin M, Chan Y (2012) Aqueous-phase reactions on hollow silica-encapsulated semiconductor nanoheterostructures. Journal of the American Chemical Society 134(21): 8754-8757.

10. Xiao M, Zhao C, Chen H, Yang B, Wang J (2012) “Ship-in-a-bottle” growth of noble metal nanostructures. Advanced Functional Materials 22(21): 4526-4532.

11. Shmakov SN, Jia Y, Pinkhassik E (2014) Selectively initiated ship-in-abottle assembly of yolk-shell nanostructures. Chemistry of Materials 26(2): 1126-1132.

12. Qiao ZA, Huo Q, Chi M, Veith GM, Binder AJ, et al. (2012) A "Ship-in-abottle" approach to synthesis of polymer dots@silica or polymer dots@ carbon core-shell nanospheres. Advanced Materials 24(45): 6017-6021.

13. Sanlés Sobrido M, Pérez Lorenzo M, Rodríguez González B, Salgueiriño V, Correa Duarte MA (2012) Highly active nanoreactors: nanomaterial encapsulation based on confined catalysis. Angewandte Chemie International Edition 51(16): 3877-3882.

14. Vasquez Y, Henkes AE, Bauer JC, Schaak RE (2008) Nanocrystal conversion chemistry: A unified and materials-general strategy for the template-based synthesis of nanocrystalline solids. Journal of the Solid State Chemistry 181(7): 1509-1523.

15. Piao Y, Kim J, Na HB, Kim D, Baek JS, et al. (2008) Wrap-bake-peel process for nanostructural transformation from- $\mathrm{FeOOH}$ nanorods to biocompatible iron oxide nanocapsules. Nature Materials 7: 242-247.

16.Zhou HP, Wu HS, Shen J, Yin AX, Sun LD, et al. (2010) Thermally stable $\mathrm{Pt} / \mathrm{CeO}_{2}$ hetero-nanocomposites with high catalytic activity. Journal of the American Chemical Society 132(14): 4998-4999.

17. Chen H, Wang D, Yu Y, Newton KA, Muller DA, et al. (2012) A surfactant-free strategy for synthesizing and processing intermetallic platinum-based nanoparticle catalysts. Journal of the American Chemical Society 134(44): 18453-18459.

18. Wang GH, Hilgert J, Richter FH, Wang F, Bongard HJ, et al. (2014) Platinum-cobalt bimetallic nanoparticles in hollow carbon nanospheres for hydrogenolysis of 5-hydroxymethylfurfural. Nature Materials 13: 293300 . 
19. Cozzoli PD, Teresa P, Manna L (2006) Synthesis, properties and perspectives of hybrid nanocrystal structures. Chemical Society Reviews 35(11): 1195-1208.

20. Shin JM, Kim HK, Lee IS (2008) Synthesis of $\mathrm{Fe}_{3} \mathrm{O}_{4} / \mathrm{PdO}$ heterodimer nanocrystals in silica nanospheres and their controllable transformation into $\mathrm{Fe}_{3} \mathrm{O}_{4} / \mathrm{Pd}$ heterodimers and FePd nanocrystals. Chemical Communications pp. 5553-5555.

21. Kim YJ, Choi JK, Lee DG, Baek KJ, Oh SH, et al. (2015) Solid-state conversion chemistry of multicomponent nanocrystals cast in a hollow silica nanosphere: morphology-controlled synthesis of hybrid nanocrystals. ACS Nano 9(11): 10719-10728.

22. Kim SH, Jeong H, Kim J, Lee IS (2015) Fabrication of supported AuPt alloy nanocrystals with enhanced electrocatalytic activity for formic acid oxidation through conversion chemistry of layer-deposited $\mathrm{Pt}^{2+}$ on $\mathrm{Au}$ nanocrystals. Small 11(37): 4884-4893.

23. Jeon KW, Lee DG, Kim YK, Baek K, Kim K, et al. (2017) Mechanistic insight into the conversion chemistry between $\mathrm{Au}-\mathrm{CuO}$ heterostructured nanocrystals confined inside $\mathrm{SiO}_{2}$ nanospheres. Chemistry of Materials 29(4): 1788-1795.
24. Koo JH, Lee SW, Park JY, Lee IS (2017) Nanospace-confined high-temperature solid-state reactions: versatile synthetic route for high-diversity pool of catalytic nanocrystals. Chemistry of Materials 29(21): 94639471.

25. Kwon T, Jeon KW, Dutta S, Lee IS (2018) Conversion chemistry of nanoscopically confined manganese silicate: solid-state route towards porous metal oxide catalyst-support. Chemistry of Materials 30(21): 8070-8078.

26. Ha TL, Kim JG, Kim SM, Lee IS (2013) Reversible and cyclical transformation between solid and hollow nanostructures in the confined reaction of manganese oxide and silica within a nano-sized sphere. Journal of the American Chemical Society 135(4): 1378-1385.

27. Shin JM, Ha TL, Lee IS (2010) Generation of hollow $\mathrm{MnSiO}_{3}$ nanostructures through the solid-state reaction of $\mathrm{Mn}_{3} \mathrm{O}_{4}$ and $\mathrm{Pd} / \mathrm{PdO}$ nanocrystals dimensionally confined within nanosized silica spheres. European Journal Inorganic Chemistry 2010(3): 357-360.

28. Kim JG, Kim SM, Lee IS (2015) Mechanistic insight into the yolk@shell transformation of MnO@Silica nanospheres incorporating $\mathrm{Ni}^{2+}$ ions toward a colloidal hollow nanoreactor. Small 11(16): 1930-1938.

For possible submissions Click below: 